
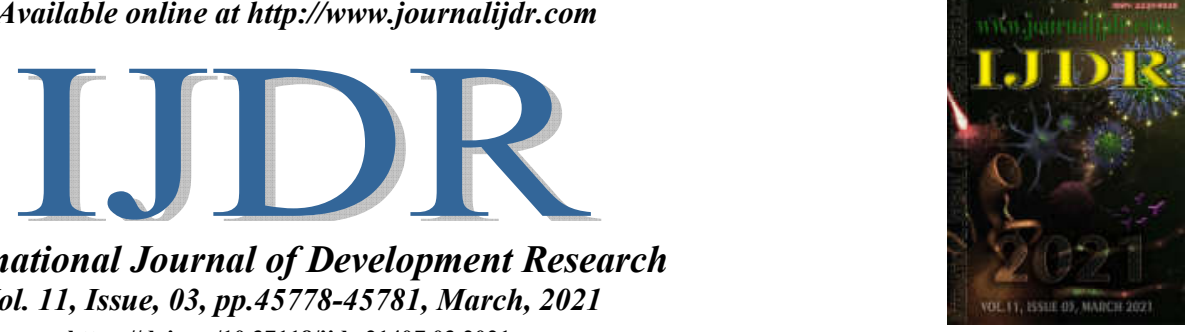

ISSN: 2230-9926

International Journal of Development Research

Vol. 11, Issue, 03, pp.45778-45781, March, 2021

https://doi.org/10.37118/ijdr.21407.03.2021

OPEN ACCESS

\title{
CONHECIMENTO E ADESÃO DE UNIVERSITÁRIAS NA PREVENÇÃO DO CÂNCER DE COLO DE ÚTERO NO INTERIOR DE SÃO PAULO - BRASIL
}

\author{
Natália Luiz Neto, Débora Cristina Carretero, Isabela de Lemos Duarte, Laís Santos \\ Nunes, Laurynês de Castro and Márcia Cristina Taveira Pucci Green
}

Universidade de Franca (UNIFRAN), Franca - SP - Brasil

\begin{tabular}{|c|c|}
\hline ARTICLE INFO & ABSTRACT \\
\hline Article History: & \multirow{3}{*}{$\begin{array}{l}\text { Objetivo: Identificar o nível de conhecimento sobre o câncer de colo uterino, as possíveis } \\
\text { limitações e dificuldades na realização da citologia oncológica e a vacinação contra o HPV de } \\
\text { universitárias da Universidade de Franca. Métodos: Foi aplicado um formulário online em alunas } \\
\text { da UNIFRAN (Universidade de Franca) pelo Google Forms após consentimento do TCLE. Os } \\
\text { dados coletados foram analisados utilizando o Excel. Resultados: Quando questionadas sobre o } \\
\text { Papanicolau } 97,4 \% \text { das participantes dizem conhecer o exame sendo que } 45,17 \% \text { foram } \\
\text { apresentadas a ele pelos profissionais de saúde. Apenas } 59,21 \% \text { sabem o verdadeiro motivo para } \\
\text { sua realização e } 111 \text { responderam a frequência correta de realização. A grande maioria das } \\
\text { participantes se encaixava em algum fator de risco para o a doença. Somente } 53 \text { mulheres } \\
\text { receberam a vacina. Conclusão: Muitos fatores levam à baixa adesão ao exame, apontado para a } \\
\text { importância da implantação de serviços e políticas públicas visando a ampliação do conhecimento } \\
\text { da população sobre o tema. }\end{array}$} \\
\hline $\begin{array}{l}\text { Received } 07^{\text {th }} \text { January, } 2021 \\
\text { Received in revised form } \\
27^{\text {th }} \text { January, } 2021 \\
\text { Accepted } 08^{\text {th }} \text { February, } 2021 \\
\text { Published online } 30^{\text {th }} \text { March, } 2021\end{array}$ & \\
\hline $\begin{array}{l}\text { Key Words: } \\
\text { Papanicolau; Rastreamento; Saúde da } \\
\text { mulher; Câncer de Colo Uterino. }\end{array}$ & \\
\hline
\end{tabular}

Copyright (C) 2021, Natália Luiz, Neto et al. This is an open access article distributed under the Creative Commons Attribution License, which permits unrestricted use, distribution, and reproduction in any medium, provided the original work is properly cited.

Citation: Natália Luiz Neto, Débora Cristina Carretero, Isabela de Lemos Duarte, Laís Santos Nunes, Laurynês de Castro and Márcia Cristina Taveira Pucci Green, 2021. "Conhecimento e adesão de universitárias na prevenção do câncer de colo de útero no interior de são paulo - brasil", International Journal of Development Research, 11, (03), 45778-45781.

\section{INTRODUCTION}

Dados do Instituto Nacional do Câncer (INCA-2019), alertam para câncer do colo do útero (CCU) como a terceira neoplasia em incidência e mortalidade entre as mulheres brasileiras, excluído o câncer de pele não melanoma. Mundialmente, possui incidência anual de 530 mil novos casos e 265 mil óbitos por ano, sendo a quarta causa de morte por câncer entre as mulheres ${ }^{(1)}$. A história natural da doença revela grande potencial de prevenção e cura em virtude desua lenta evolução, passando por vários estágios de lesões intra-epiteliais pré-cancerosas antes de chegar à forma invasiva, permitindo que a doença seja detectada ainda nos estágios iniciais ${ }^{(1)}$. A detecção precoce através da Citologia Oncótica $(\mathrm{CO})$ e a vacinação contra o Papiloma Vírus Humano (HPV) são medidas protetivas eficazes, podendo proteger pacientes virgens dos tipos de HPV, 6,11,16 e 18 contidos nas vacinas. $\mathrm{O}$ exame de rastreamento, popularmente conhecido como exame de Papanicolau ou citologia oncológica (CO), assim como a citologia em base líquida, são capazes de identificação de células de lesões precursoras do câncer cervical. No Brasil, seu rastreamento é recomendado a partir dos 25 anos, para mulheres que já iniciaram atividade sexual, e interrompido aos 64 anos, visto que, após essa idade, com resultados normais, o risco de desenvolvimento do câncer é irrelevante ${ }^{(2)}$, não justificando exposição desta faixa etária ao rastreamento.
A aplicação do exame é reconhecida mundialmente como uma estratégia segura e eficiente para diagnósticos precoces, atingindo prognósticos favoráveis, com capacidade de curapróximo a $100 \%{ }^{(2)}$. No entanto, mesmo tendo sido introduzido no Brasil na década de 1950 , cerca de $40 \%$ das mulheres inclusas na faixa etária de rastreio nunca realizaram o teste de Papanicolau ${ }^{(3)}$. Ademais, no rastreio oportunístico ocorrido no Brasil, no qual as mulheres participam de acordo com a busca espontânea pelo serviço, há uma maior procura por mulheres fora da população alvo em detrimento daquelas inclusas no grupo etário, levando a uma baixa cobertura efetiva ${ }^{(4)}$. Segundo a OMS (Organização Mundial da Saúde), cerca de $80 \%$ da mortalidade por câncer cervical poderia ser reduzida pelo rastreamento de mulheres entre 25 e 60 anos por meiodo teste e acompanhamento adequado das lesões encontradas, o que depende não só da eficácia da rede de saúde, mas principalmente da colaboração consciente dessa população ${ }^{(5)}$. Alguns fatores associados à baixa procura pelo exame de citologia oncótica estão a idade avançada, o baixo nível socioeconômico e de escolaridade, determinadas crenças e não possuir parceiro fixo ${ }^{(6)}$. A presença do HPV, um vírus intracelular com mais de 100 tipos existentes, é o principal fator de risco para atipias da mucosa uterina, sendo responsável por $99 \%$ dos casos de neoplasia nesta região ${ }^{(7)}$. A vacina disponível no Sistema Único de Saúde (SUS) protege contra o HPV de baixo risco (tipos 6 e 11, que causam verrugas ano-genitais) e de alto risco (tipos 16 e 18, responsável pelo câncer de colo uterino) ${ }^{(8)}$. A população-alvo prioritária são meninas 
na faixa etária de 9 a 14 anos, meninos de 11 a 14 anos e mulheres vivendo com HIV na faixa de 9 a 26 anos ${ }^{(8)}$. A vacina tem sido utilizada como método promissor quanto à segurança, eficácia e durabilidade de resposta imunológica, garantido a prevenção da doença ${ }^{(9)}$. Além da colpocitologia oncótica é possível identificar o vírus através de outros métodos como a Captura híbrida, citologia em base liquida, biomarcadores p16 e o Ki-67 hibridização in situ e Coteste. Os testes moleculares possibilitam a detecção do HPV, identificação do tipo e monitorização ${ }^{(10)}$. Diante do exposto visou-se identificar a relação entre a realização do Papanicolau e fatores comportamentais, características sociodemográficas, além da atividade sexual e reprodutiva, que implicam possíveis barreiras para a execução do exame e da vacinação da população jovem.

\section{MÉTODOS}

O estudo em questão tem caráter epidemiológico quantitativo, do tipo transversal, que visa identificação de possíveis motivos para a não adesão à coleta da Colpocitologia Oncótica (Papanicolau). Para isso, foi realizado um formulário respondido por estudantes que atenderam os critérios de inclusão desta pesquisa: universitárias da Universidade de Franca (UNIFRAN), acima de 18 anos, que concordaram e consentiram com o TCLE. Assim, os critérios de exclusão foram menores de 18 anos, universitárias que não pertencem à UNIFRAN ou não desejaram participar da pesquisa. $O$ formulário foi confeccionado a partir do aplicativo de gerenciamento Google Forms, acessado gratuitamente pelo Google, o qual permite uma prática coleta de informações, transferindo-as automaticamente para uma planilha do mesmo programa. A aplicação do questionário realizada via email, enviado aleatoriamente para 378 alunas da UNIFRAN, incluindo diferentes áreas do conhecimento, sendo estas representadas pela área da Saúde, Ciências Exatas e Humanas, visando alcançar um $\mathrm{N}=200$. Durante o período de 08/03/2021 e 10/03/2021, atingiu-se 234 respostas, número superior ao esperado. É importante lembrar que, todos os membros deste estudo, concordaram em participardo mesmo assinando o Termo de Consentimento Livre e Esclarecido (TCLE) presente no respectivo formulário.

Ao optarem pela opção "prosseguir" a participante concordou com a participação na pesquisa, sendo direcionada ao formulário para coleta de dados. Este consiste em um modelo, o qual foi transcrito de forma integral para a plataforma Google, de autoria própria, elaborado com base em pesquisas de plataformas online, como BibliotecaVirtual em Saúde, SCIELO, Pubmed, entre outros, selecionando aqueles de maior relevância, de acordo com o tema em pauta, com os seguintes descritores: Câncer do Colo do Útero, Papanicolau e adesão ao exame de Papanicolau. Após encerrada a coleta de dados, esses foram transferidos à uma planilha do Excel, onde tornou-se possíveis através das ferramentas disponíveis neste software o estudo e correlação das variáveis a serem analisadas de acordo com os objetivos propostos pelo trabalho. Os benefícios esperados com o presente estudo são o fomento de informações e questionamentos sobre o câncer de colo e a possibilidade de ampliar o conhecimento dos entraves para a realização da prevenção e detecção das lesões precursoras do câncer de colo do útero. Não foram previstos riscos graves, entretanto, é possível que ao responderem o questionário, a participante tenha se sentido desconfortável, constrangida ou ter alguma lembrança desagradável.

Para minimizá-lo, o questionário foi aplicado individualmente para que a participante se sinta à vontade. A invasão de privacidade ao responder a questões com tema sexualidade foi reduzido pelo anonimato do questionário, o qual poderia ser preenchido apenas com as iniciais das participantes; a identidade e todas as informações cedidas no questionário foram mantidos em sigilo absoluto sob responsabilidade do pesquisador. Ainda, é possível vazamento não intencional dos dados coletados, por condições alheias aos cuidados dos participantes deste estudo, sendo este risco evitado quando as informações são guardadas por somente um autor, evitando o repasse de informações para os demais autores.

\section{RESULTADOS}

O estudo contou com participantes de diversas áreas do conhecimento, representadas pela área da Saúde, sendo elas 169 estudantes $(72,22 \%)$, versus demais áreas $(27,77 \%)$, incluindo ciências exatas e humanas, somando 65 respostas. Os cursos da área da saúde foram representados por: Medicina (62 alunas), Psicologia (26), Odontologia (23), Fisioterapia (16), Enfermagem (14), Biomedicina (10), Farmácia (5), Medicina Veterinária (4), Biologia (4), Educação Física (2), Estética (2) e Nutrição (1). As outras esferas foram compostas por: Engenharia (20), Pedagogia (17), Administração (15), Direito (11), Ciências Contábeis (1) e Arquitetura (1). Em relação às idades dos membros desta pesquisa, 199 possuem entre 18-25 anos; 25 têm entre 26-30 anos; 4 mulheres têm de 31 a 35 anos; 3 participantes, entre $36-40$ anos e 3 , de 46-50 anos. Analisando os dados obtidos, nota-se que quando questionado sobre o Papanicolau, 228 mulheres $(97,4 \%)$ responderam SIM quanto ao conhecimento deste exame, enquanto 6 mulheres responderam NÃO (2,6\%). Diante daquelas que possuem conhecimento sobre o exame, a maioria o obteve através dos profissionais da saúde (45,17\%), seguidos pelos familiares $(39,91 \%)$, escola $(8,33 \%)$, com amigas, rádio/tv/jornal e "outros" somando $6,59 \%$. Ainda em relação às 228 mulheres que "conhecem" o Papanicolau, somente 157 informaram já ter realizado o exame antes, o que corresponde a uma taxa de $31,14 \%$ de desaprovação a Colpocitologia Oncótica.

Surpreendentemente, apenas 135 (59,21\%) mulheres que afirmam ter conhecimento sobre o assunto, sabem o verdadeiro motivo para realização do exame, tendo respondido adequadamente: prevenir o câncer de colo útero. Cinquenta e uma (51) delas responderam ser "porque toda mulher precisa fazer" sem ter assinalado o real motivo para isso; semelhantemente 38 o fizeram por orientação do profissional de saúde e 4 delas, realizaram o exame devido orientação de familiares e/ou amigos. Somente 111 universitárias responderam devidamente a frequência de realização do Papanicolau: uma vez ao ano, dessas, $100 \%$ informaram ter realizado o último exame no ano de 2019 ou 2020. Contudo, nota-se que 51,31\% (123) daquelas que dizem conhecer o exame, não sabem a periodicidade para realiza-lo. Quando retiradas aquelas que afirmaram não ter conhecimento ou nunca ter realizado o exame anteriormente (75 mulheres), este valor cai para $21,05 \%$, embora, seja ainda elevada. Levando em conta a presença de atividade sexual prévia - 212 mulheres - $37(17,45 \%)$ rã fazem acompanhamento com um profissional ginecologista, enquanto 47 delas nunca realizaram Papanicolau anteriormente, o que equivale $22,16 \%$. Considerando a idade das pacientes, dentre as 35 universitárias que possuem idade superior a 25 anos, 3 delas $(8,57 \%)$ ainda não realizaram o exame. Com exceção daquelas que nunca tiveram relação sexual previamente (19), o uso do preservativo foi citado por 126 alunas, enquanto 89 delas informaram não fazer uso, sendo que dessas, 35 relatam nunca o utilizar. Atentando àquelas que utilizam o preservativo, 73 mulheres dizem "sempre" utilizarem, 41 responderam "às vezes" e 14, "raramente". Quando analisado o estado civil destas universitárias, somente 8 mulheres, as quais nunca utilizam preservativo, são casadas ou possuem relação estável, correspondendo a $22,85 \%$, enquanto 27 são solteiras $(77,14 \%)$. Destas, 23 possuem parceiro fixo.

Acerca do histórico positivo de Infecção Sexualmente Transmissível (IST) 11 mulheres responderam que SIM. Meio a estas, a sexarca com 15 anos ou menos ocorreu em 5 alunas $(45,45 \%)$. Três (3) mulheres informaram ter tido 3 ou mais parceiros sexuais no último ano enquanto 8 delas possuíam parceiro fixo. A vacina só foi administrada a 5 participantes com história previa de IST e 2 ainda não tiveram acesso ao exame preventivo. A principal dificuldade para realização deste, relatada pelas pacientes com IST, se resumem a "Falta de Tempo" (54,54\%), "Trauma por exame anterior" (18,18\%), "Vergonha/Medo" (18,18\%) ou nenhuma dificuldade (9,09\%). Em 56 entrevistadas, a primeira relação sexual ocorreu com $\leq 15$ anos, correspondendoa $23,9 \%$; 70 mulheres $(29,9 \%)$ afirmam que no último ano tiveram no mínimo 3 parceiros e $159(68,8 \%)$ não fazem uso adequado do preservativo em todo ato sexual. Dentre as mulheres que 
já tiveram relação sexual, 75 participantes não possuem parceiro fixo e apenas 30 destas (40\%) fazem o exame anualmente, como preconizado. Quanto à vacinação 124 mulheres $(53 \%)$ disseram ter recebido a vacina e $110(47 \%)$ não receberam. Destas últimas, apenas 12 delas não manifestaram o desejo de recebê-la. Alémdisso, 87 (79\%) das universitárias não vacinadas estão na faixa etária de 18-25 anos; 17 (15,5\%), estão entre 26-30 anos; 2 (1,8\%), entre 31-35 anos; 1 (1\%) aluna tem entre $36-40$ anos e $3(2,7 \%)$, entre $46-50$ anos. Ao correlacionar os motivos de maior dificuldade para realizar o exame com a idade, dentre aquelas que justificaram a Vergonha/medo, 79 delas possuem entre 18-25 anos; 3 entre 26-30 anos; 1 entre 31- 35 anos e 3 entre 46-50 anos. As que justificaram ser por falta de tempo, 61 possuem entre 18-25 anos; 6 entre 26-30 anos; 1 entre 31-35 anos e 2 têm entre 36-40 anos. 14 participantes informam que o trauma por exame anterior/desconforto foi a principal dificuldade para realizar o exame têm entre 18-25 anos e 3, entre 26-30 anos. Das que responderam ser a falta de acesso, 6 estão entre 18-25 anos; 2 entre 26-30 anos e 1, entre 31-35 anos. Aquelas cujo principal obstáculo é "o profissional médico ser homem", 7 estão entre 18-25 anos e 1, entre 31-35 anos. Quatro (4) integrantes da amostra não sabem a necessidade de realizar o exame, todas tendo entre 18-25 anos. As que usaram a religião como justificativa, 4 possuem entre 18-25 anos e 1, entre 2630 anos. Ainda houve aquelas que se justificaram assinalando o grupo "outros", o qual envolve tanto as que não realizaram o exame por não terem iniciado a vida sexual, quanto aquelas que não possuem nenhuma dificuldade. Destas, 26 possuem entre 18-25 anos; 6 têm entre 26-30 anos e 1, entre 31-35 anos. Considerando o nível socioeconômico, 99 possuem de 1-3 salários mínimos. Destas, 29 $(29,29 \%)$ não fazem acompanhamento com médico especialista, enquanto $38(38,38 \%)$ NUNCA realizaram o exame preventivo anteriormente, com exceção daquelas em que a atividade sexualainda não ocorreu (7 mulheres). Oitenta e duas (82) alunas convivem com 4-7 salários, sendo que $16(19,51 \%)$ delas não vão frequentemente ao ginecologista e $22(26,82 \%)$ ainda não realizaram o Papanicolau, excluídas as 4 que não possuem vida sexual. Dentre as 53 universitárias que vivem com mais de 8 salários mínimo, 4 (7,54\%) não acompanham com o ginecologista e $4(7,54 \%)$ delas ainda não fizeram o exame anteriormente, restringindo as 3 participantes sem relação sexual prévia. Observa-se que, das 169 mulheres estudantes da área da saúde, $35(20,7 \%)$ delas não possuem a devida periodicidade nas consultas com o ginecologista. Meio a estas, 28 (80\%) mulheres já tiveram relações sexuais. Já em relação às outras áreas do conhecimento (65 respostas), 15 mulheres não fazem acompanhamento ginecológico e destas, 9 já alcançaram a sexarca.

\section{DISCUSSÃO}

No estudo em questão $97,4 \%$ das mulheres disseram conhecer a existência do Papanicolau, resultado semelhante ao encontrado em Santa Maria - RS, onde $70 \%$ das participantes teriam o mesmo conhecimento ${ }^{(11)}$. Ao serem questionadas sobre o motivo para a realização do exame $59,21 \%$ responderam adequadamente sobre a prevenção do câncer de colo de útero, concordando com os resultados encontrados em um estudo realizado no município de São José de Ribamar-MA, no qual a maioria das participantes também possuíam tal entendimento ${ }^{(12)}$. Apesar de representarem a maioria do grupo, a sensibilização e educação em saúde ainda de fazem essenciais como meio de orientação e empoderamento, visto que 93 delas escolheram entre as outras opções possíveis sem ainda considerar a resposta mais adequada. Dessa forma é possível ainda vincular que apesar de realizarem o exame muitas não sabem o intuito e a importância do mesmo. Apesar do alto índice de conhecimento do exame, 51,31\% delas não sabem a periodicidade correta de sua realização, que se refere ao intervalo de um ano conforme preconizado pela OMS, sendo que após dois exames normais consecutivos o exame pode ser realizado em esquema trienal ${ }^{(13)}$. Entre aquelas que responderam a frequência correta todas alegaram ter feito o exame no ano de 2019 ou 2020, sendo a realização do mesmo imprescindível para intervenção prévia ao agravo da doença e diagnóstico precoce, o que possibilita o tratamento em estágios iniciais ediminuição das taxas de incidência da doença e suas complicações ${ }^{(14)}$.
Neste contexto de prevenção secundária, o exame citopatológico é essencial como rastreio, visando a redução da progressão para o câncer. Quando questionadas sobre a realização do Papanicolau, felizmente a minoria das mulheres com 25 anos ou mais responderam nunca ter feito o mesmo. Estudos apontam que esse grupo, o qual nunca realizou o screening, desenvolve o câncer de colo com maior frequência, visto a não adesão preventiva. Este resultado positivo corrobora com a redução dos índices de morbimortalidade pela neoplasia desde o início da introdução do programa de rastreamento (14). O uso do preservativo foi citado pela maioria das participantes, porém um número significativo descreveu não o utilizar sempre. $77,14 \%$ das mulheres que não fazem uso de camisinha são solteiras, de tal forma, esse comportamento as torna mais suscetíveis a infecções sexualmente transmissíveis conforme demostra vários estudos dentre ele o realizado em Ribeirão Pires - São Paulo. Vale ainda ressaltar que entre elas há uma porcentagem que não possuem parceiro fixo, mesmo com vida sexual ativa ${ }^{(15)}$.

Destas que nunca fazem uso do método de barreira, $22,85 \%$ são casadas ou em união estável, esse comportamento pode ser reflexo da confiança depositada em seus parceiros e posição de desvantagem no momento da escolha do uso do preservativo, na maioria das vezes. Ademais, essas mulheres também compõem um grupo de maior vulnerabilidade em relação a Infecções Sexualmente Transmissíveis (ISTs) ${ }^{(16)}$. Sendo a infecção prévia pelo papiloma vírus humano (HPV) o principal fator de risco parao desenvolvimento da neoplasia invasiva do colo, comportamentos como sexarca precoce, a multiplicidade de parceiros e a ausência do uso do preservativo como método de barreira e a não adesão ao exame preventivo são importantes representantes de risco para o câncer. Correlacionando os dados da literatura com os encontrados no presente trabalho foi observado que um grande número de mulheres que se encaixam em um ou mais dos fatores de risco discutidos visto que $22 \%$ dessas nunca realizaram o exame ginecológico sendo apenas $8,57 \%$ não possuíam idade acima de 25 anos como preconizado pela OMS. Entre as sexualmente ativas que não possuem parceiro fixo e apenas (40\%) fazem o exame anualmente um número inadequado para a prevenção de doenças. Ao se expor em relações sexuais desprotegidas, essas mulheres estão sujeitas a se infectar não somente com o HPV, mas com vírus de outras ISTs. Neste cenário, o exame citopatológico apresenta-se novamente como medida de detecção inicial de lesões papilomatosas ainda passivas de tratamento ${ }^{(14)}$. No atual estudo, a maioria das participantes alegou ter recebido a vacina contra o HPV (53\%), e daquelas não imunizadas $89 \%$ demonstraram interesse em receber a imunização. Estes resultados são corroborados por uma Revisão Integrativa da Literatura sendo observado que as adolescentes se envolvem mais na decisão de receber a vacina a partir dos 14 anos, quando já iniciaram sua vida sexual e possuem um relacionamento (17). Ademais, o conhecimento sobre a correlação do vírus com o câncer do colo de útero é essencial na adesão à vacina.

Sabe-se que a cobertura preconizada do Papanicolau ainda está distante de ser atingida no país, algo refletido neste estudo, que evidencia alguns dos principais impedimentos à realização do exame. A maior parte das estudantes descreveu a "vergonha/medo" como barreira, este sentimento é também um dos mais recorrentes em outros estudos, como descrito no Estudo Descritivo Exploratório Qualitativo realizado em Vitória da Conquista (BA). Neste contexto estão envolvidos receios como a exposição do corpo e padrões sociais precursores que envolvem a religião, por exemplo, citada como dificuldade por quatro participantes. A falta do conhecimento integral da necessidade do exame corrobora para o aumento de ideias preconcebidas, da ansiedade e do medo ${ }^{(18)}$. O trauma anterior e desconforto também tiveram uma pontuação significativa, evidenciando o quanto a transferência de vivências pregressas não exitosas pode refletir no comportamento e expectativas das pacientes, dificultando a construção de uma relação profissional efetiva, como citado na publicação Relação Médico Paciente em $2003{ }^{(19)}$. Um estudo realizado em uma ESF (Estratégia da Saúde da Família) de Ribeirão Preto, São Paulo, ressalta o quanto o vínculo é essencial na união da autonomia das usuárias e responsabilidade profissional ${ }^{(20)}$. Os cuidados profissionais durante o exame, assim como sua postura e 
acolhimento são fundamentais para a impressão de segurança e conforto da mulher, algumas ainda apontaram oprofissional do sexo masculino como um impedimento. Mesmo que em menor número neste estudo, isso reflete padrões de gênero enraizados na sociedade, nos quais a sexualidade, exposição da genitália feminina e manipulação da mesma ainda são consideradas, por vezes, imorais, principalmente quando o médico é homem, discussão também presente no descrito estudo realizado em Vitória da Conquista ${ }^{(18)}$.

A falta de tempo para realização do exame teve grande destaque como obstáculo à realização do mesmo, mostrando um descuido com a saúde que muitas vezes é refletido numa busca tardia pelo atendimento. Essa constatação reforça a superioridade do tratamento em detrimento da prevenção, influência do padrão biomédico ainda vigente em nosso meio ${ }^{(18)}$. A falta de acesso ao procedimento não parece ser uma realidade das estudantes em questão, sendo escolhida por uma minoria. Porém, estudos demonstram que mulheres com menor acessibilidade a programas de saúde são as que possuem maior risco para o desenvolvimento de lesões cervicais, já que a etapa preventiva é inexistente ou depende se serviços privados, por vezes inacessíveis ${ }^{(18)}$.

\section{CONCLUSÃO}

Muitos fatores levam à baixa adesão ao exame de Colpocitologia Oncótica, ademais umaparcela significativa da população que realiza periodicamente o exame preventivo ainda desconhece seu real objetivo, mesmo estando no meio universitário, onde presume-se que o esclarecimento e a difusão de informação sejam maiores que na população geral. A falta de conhecimento e imposição de valores preconcebidos tornam o medo e a vergonha grandes obstáculos à realização do exame. Dessa forma ressalta-se a importância da implantação de serviços e políticas públicas que objetivem um efetivo empoderamento das mulheres com relação a sua saúde, a partir de estratégias de comunicação que tornem a informação clara e de fácil acesso. Assim, a ação dos profissionais da saúde e o estabelecimento de vínculos com suaspacientes se fazem essenciais, auxiliando na quebra de preconceitos ainda existentes acerca dasaúde feminina.

\section{REFERÊNCIAS}

Saúde Md. Instituto Nacional do Câncer. [Online].; 2019. Available from: https://www.inca.gov.br/controle-do-cancer-do-colo-doutero/conceito-e-magnitude.

Saúde Md. Diretrizes Brasileiras para o Rastreio do Câncer do Colo do Utero. 26th ed. Cancer INd, editor. Rio de Janeiro: Divisão de Apoio à Rede de Atenção Oncológica; 2011.

Batista B.P.R.; Mastroeni F.M. ACTA Paulista de Enfermagem. [Online].; 2012. Available from: http://www.scielo.br/pdf/ape/ v25n6/v25n6a09.pdf.

Speck G.M.N; Carvalho P.J. [Documento - Dossiê de estratégias do câncer de colo uterino no Brasil FEBRASGO].; 2017.
Silva M.A.; Freitas H.G.; Ribeiro R.L.; Oliveira M.N.L.; Sanches F.C.A.; Thuler L.C.S. Fatores que, na Visão da Mulher, Interferem no Diagnóstico Precoce do Câncer do Colo do Útero. Revista Brasileira de Cancerologia. 2018; 64(1).

Aguiar P.M.; Stock G.T.; Lopes G.D.L.;Almeida M.S.; Tadokoro H.; Gutierres B.S.; et al. Perfil de mulheres que realizam o exame de prevenção de câncer cérvico uterino em um centro especializado a saúde da mulher. Revista Digital EFDeportes.com. 2013; 17.

Janete Tamani Tomiyoshi Nakagawa JSMB. Vírus HPV e câncer de colo de útero. Revista Brasileira de Enfermagem. 2010 março; $3(63)$.

Saúde Md. Informe técnico da ampliação da oferta das vacinas papilomavírus humano 6,11,16,18 (recombinante)- vacina HPV quadrivalente e meningocócica C (conjugada). 2018..

Saúde Md. Ministerio da Saúde. [Online].; 2019. Available from: http://portalms.saude.gov.br/saude-de-a-z/hpv.

Kamila Mtos de Albuquerque PGF, Andrade CLTd. Cobertura do teste de Papanocolaou e fatores associados à não-realização: um olhar sobre o Programa de Prevenção do Câncer do Colo do Útero em Pernambuco, Brasil. Caderno de Saúde Pública. 2009 março; 2(25).

Rocha D.B.; Bisognin P.; Cortes L. F.; et al. Exame de Papanicolau: conhecimento de usuárias de uma unidade básica de saúde. Revista de Enfermagem da UFSM. 2012 Set/Dez;(3).

Oliveira A.F.; Cunha C.L.F.; Viégas I.F. et al. Estudo sobre a adesão ao exame citopatológico de papanicolau em um grupo de mulheres. Revista de Pesquisa em Saúde. 2010 jan/abr; 111(1).

NATIONAL CANCER INSTITUTE - INCA. [Online].; 2020. Available from: https://www.inca.gov.br/en/node/1194.

Rangel G.; Lima L.D.; Vargas E.P. Condicionantes do diagnóstico tardio do câncer cervical na ótica das mulheres atendidas no Inca. SAÚDE DEBATE. 2015 out/dez; 39.

Machado L.S; Pires M.C. Rastreamento do papilomavírus humano (HPV) através do exame de papanicolau. Revista enfermagem atual. 2018;(85).

Carreno I.; Costa J.C.D. Uso de preservativos nas relações sexuais: estudo de base populacional. Revista de Saúde Pública. 2006; 40(4).

A.M.C. C, E.M.L.R. A, al NLTe. Adesão à vacina HPV entre os adolescentes: revisão integrativa. Texto \& Contexto Enfermagem. 2019; 28(20).

Aguilar R. P.; Soares D.A. Barreiras à realização do exame Papanicolau: perspectivas de usuárias e profissionais da Estratégia de Saúde da Família da cidade de Vitória da Conquista - BA. Revista de Saúde Coletiva. 2015;(2).

S. S. Relacionamento médico-paciente. Revista Brasileira de Reumatologia. 2003 jul/ago; 43(4).

Oliveira M. M.; Pinto I.C. Percepção das usuárias sobre as ações de Prevenção do Câncer do Colo do Útero na Estratégia Saúde da Família em uma Distrital de Saúde do Município de Ribeirão Preto,São Paulo,Brasil. Revista Brasileira de Saúde Materno Infantil. $2007 \mathrm{jan} / \mathrm{mar}$; 7(1). 